\title{
Experimental and Theoretical Verification of Impact Response on Air Thrust Bearing with Topological Optimized Groove
}

\author{
Masayuki Ochiai ${ }^{1)^{*}}$, Hayato Sasaki ${ }^{2)^{*}}$, Yuta Sunami ${ }^{1)}$ and Hiromu Hashimoto ${ }^{1)}$ \\ ${ }^{1)}$ Department of Mechanical Engineering, Tokai University \\ 4-1-1 Kitakaname, Hiratsuka-shi, Kanagawa 259-1292, Japan \\ ${ }^{2)}$ School of Engineering, Corse of Mechanical Engineering, Graduate School of Tokai University \\ 4-1-1 Kitakaname, Hiratsuka -shi, Kanagawa 259-1292, Japan \\ "Corresponding author: ochiaim@keyaki.cc.u-tokai.ac.jp
}

( Manuscript received 22 February 2014; accepted 24 June 2014; published 15 April 2015 )

( Presented at Technical Session in the $5^{\text {th }}$ World Tribology Congress TORINO September 2013 )

\begin{abstract}
This paper describes experimental and theoretical verification in impact response of optimized grooved bearing designed by topological optimization. Firstly, in order to measure the impact response characteristics accurately temperature compensation method for air film thickness of bearing is proposed and confirmed by experiment of bearing reaction force with air film thickness. Then, in this study, impact experiments were conducted with the optimized bearing and spiral grooved bearing and compared with theories of linear and non-linear analyses. As a result, the optimized bearing showed the high impact resistance compared with spiral grooved bearing. In addition, it was confirmed that the results of non-linear analyses are good agreement with those of experiment. Finally, based on above results, the impact response calculations of both bearings by non-liner theory under more high impact force condition were conducted. As a result, it is found that the response of spiral grooved bearing generates the self excited vibration. On the other hand, the response of optimized bearing showed damping waveform with time even under the high impact force conditions.
\end{abstract}

Keywords: thrust air bearing, stiffness, bearing reaction force, film thickness, vibration characteristic, experiment

\section{Introduction}

Hydrodynamic thrust air bearings have a lot of advantages such as low environmental load, low frictional loss and maintenance-free. Therefore, hydrodynamic thrust air bearings are extremely effective against saving of energy gain recognition in today's machine industry. The study related to hydrodynamic thrust air bearings has been previously performed by a lot of researchers [1-7]. On the other hand, bearing stiffness is considerably low compared with oil bearing because the viscosity of air is very low. Under these background, Hashimoto proposed an optimization method of groove geometry and carried out maximization of bearing stiffness. As a result, a new groove geometry having a bending curve in outer vicinity of the bearing surface was obtained $[8,9]$, and it was verified that the bearing has high stiffness compared with typical other bearings such as spiral grooved bearing and herring bone grooved bearing by theory and experiment. Moreover, it was confirmed that the bearing stiffness of optimized thrust air bearing is improved through appropriately suppresses the air film thickness caused by generating negative pressure on the outer circumference and positive pressure on the inner circumference of the bearing surface [8-10]. However, it has not been verified that the optimized bearing has high impact resistance against the external force. High impact resistance is being demanded to expand use of hydrodynamic thrust air bearings in many applications.

Consequently, in this study, we carried out impact experiments of the optimized bearing and spiral grooved bearing and compared with results of linear and non-linear analyses for validation.

\section{Experimental apparatus and method}

\subsection{Experimental apparatus}

Figure 1 shows a schematic diagram of experimental apparatus. The experimental apparatus consists of a stator, a test bearing and a rotor. The stator is supported in the radial direction by a hydrostatic air bearing to provide its one-degree-of-freedom operation under very 


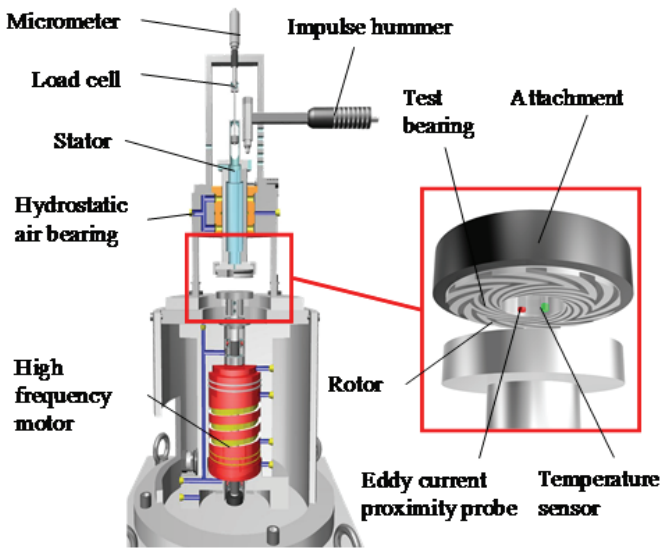

Fig. 1 Schematic diagram of experimental apparatus

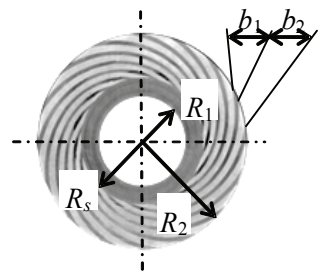

(a) Spiral grooved bearing

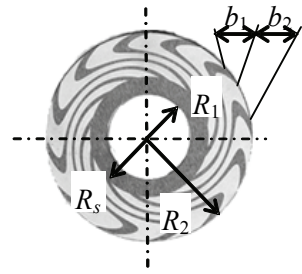

(b) Optimized bearing
Fig. 2 Groove geometries and specifications of test bearings

low friction. The test bearing can be easily replaced through an attachment. In addition, the rotational speed of shaft can be controlled from 5,500 rpm to $35,000 \mathrm{rpm}$ by a high frequency motor. Figure 2 shows groove geometries of the optimized bearing and the spiral grooved bearing which were used to experiments. The optimized bearing has novel groove geometry that was bent toward the outer circumference in which negative pressure is generated $[8,10]$. Table 1 shows the specifications of the optimized bearing and spiral grooved bearing.

Table 1 Bearing specifications

\begin{tabular}{l|c|c}
\hline Parameters & $\begin{array}{c}\text { Spiral } \\
\text { grooved }\end{array}$ & Optimized \\
\hline \hline Number of grooves $N$ & 16 & 12 \\
\hline Groove depth $h_{\mathrm{g}}, \mu \mathrm{m}$ & 21.0 & 20.0 \\
\hline $\begin{array}{l}\text { Groove width ratio } \\
\alpha=\left(b_{2} /\left(b_{1}+b_{2}\right)\right)\end{array}$ & 0.636 & 0.58 \\
\hline Seal ratio $r_{s}=\left(R_{s} / R_{2}\right)$ & 0.58 & 0.53 \\
\hline Outer radius $\quad R_{1}, \mathrm{~mm}$ & \multicolumn{2}{|c}{32.0} \\
\hline Inner radius $\quad R_{2}, \mathrm{~mm}$ & \multicolumn{2}{|c}{12.8} \\
\hline
\end{tabular}

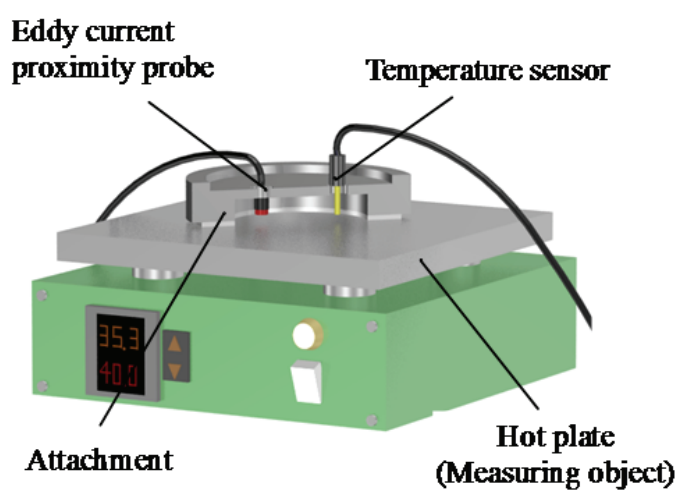

Fig. 3 Schematic diagram of experimental apparatus for environmental test

\subsection{Experimental method}

\subsubsection{Environmental test}

In this study, it is the most important to measure the air film thickness accurately. The air film thickness between the rotor and the bearing is measured by using an eddy current proximity probe as shown in Fig. 1 . However, it is considered that the probe is influenced by the effect of heat generation of air film according to shear force and a high frequency motor. As a result, large measurement errors of film thickness occur. In this study, a measurement method with the consideration of the temperature change of surrounding the eddy current proximity probe is proposed to solve this problem. Therefore, an environmental test was conducted to investigate the relationship between the temperature change and output voltage of the eddy current proximity probe.

The experimental apparatus for the environmental test used in this study is schematically illustrated in Fig. 3. The experimental apparatus consists of a hot plate, an attachment with the eddy current proximity probe and a temperature sensor. In the environmental test, the distance between the eddy current proximity probe and the hot plate was constant, and then temperature of

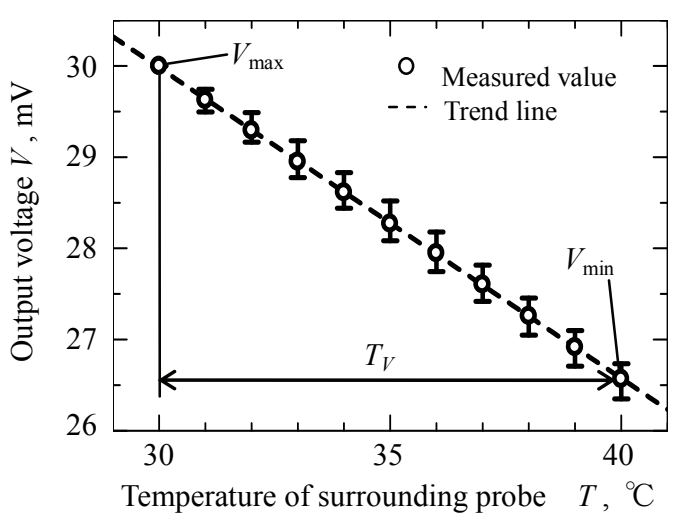

Fig. 4 Relationship between output voltage and temperature of surrounding probe 
surrounding the eddy current proximity probe was changed using the hot plate. The temperature of surrounding the eddy current proximity probe can be controlled every $0.1^{\circ} \mathrm{C}$.

Figure 4 shows the relationship between output voltage and temperature of surrounding the probe. In this study, the measurement range of the temperature were defined as 30 to $40^{\circ} \mathrm{C}$ from preliminary temperature measurement of the surrounding the probe on the experimental apparatus shown in Fig. 1. The experimental repeatability was confirmed by the measurements of more than five times. As understood from the figure, output voltage of the eddy current proximity probe is in proportion to temperature of surrounding the probe. The proportional coefficient for temperature compensation can be obtained using the following equation.

$$
\delta_{V}=\frac{\left(V_{\max }-V_{\min }\right)}{T_{V}}
$$

where $V_{\max }$ : the maximum voltage, $V_{\min }$ : the minimum voltage, and $T_{V}$ : the amount of temperature variables during calibration. The amount of output voltage of the eddy current proximity probe was measured at several temperatures and the compensation values were then added to the measurement results of film thickness. The formula is shown as follows.

$$
h_{r}=\frac{\left(V_{\text {exp. }}-V_{0}\right)}{G}+\frac{\Delta T \times \delta_{V}}{G}
$$

where $V_{\text {exp }}$ : the measured voltage, $V_{0}$ : the reference voltage, $\Delta T:$ the temperature change and $G$ : the calibration coefficient.

In this study, the value of $\delta_{v}$ and $G$ were defined as $2.8 \mathrm{mV} /{ }^{\circ} \mathrm{C}$ and $3.3 \mathrm{mV} / \mu \mathrm{m}$ respectively by the environmental test. Hence, the experimental value of film thickness is compensated by approximately $8.5 \mu \mathrm{m}$ when the temperature varies by $10 /{ }^{\circ} \mathrm{C}$.

\subsubsection{Measurement method for bearing reaction force}

Before measurement, the parallelism was checked by measuring the clearance between test bearing surface and runner surface at 12 points in the circumferential direction with the same intervals. We have been confirmed that the parallelism is $5.0 \mu \mathrm{m}$ or less.

Bearing reaction force is measured by using load cell which is installed between the micrometer and the stator as shown in Fig. 1. Test bearing is brought close to the rotating rotor using the micrometer, and then the bearing reaction force can be measured by generating pressure on the bearing surface. In this study, the load by the stator is $15 \mathrm{~N}$.

In the experiment, firstly the rotational speed of rotor was raised to the target speed. The initial air film thickness between the bearing and rotor surface was fixed in $30 \mu \mathrm{m}$ using the micrometer. And then, the test bearing was brought close to the rotor with every $2.0 \mu \mathrm{m}$ until floating of test bearing, and the bearing reaction force for each air film thickness is measured. In addition, rotational speed of shaft was fixed to $30,000 \mathrm{rpm}$.

\subsubsection{Impact experiment}

In the impact experiment, external force on the stator is given by impulse hummer, which an acceleration pickup sensor is built into. The range of external force by impulse hummer is from $15 \mathrm{~N}$ to $60 \mathrm{~N}$. And response displacement is measured by the eddy current proximity probe installed inside the attachment. In addition, the rotational speed of shaft was fixed to $35,000 \mathrm{rpm}$. The eddy current proximity probe and the impulse hammer are connected to the FFT analyzer. Output signal from the impulse hammer was used as trigger, and then response displacement of the test bearing was measured. The experiments were conducted more than ten times.

\section{Results and discussion}

\subsection{Results of bearing reaction force against air film} thickness

Figures 5(a), (b) show the relationship between bearing reaction force and film thickness. Vertical and horizontal axes on the figure show the bearing reaction

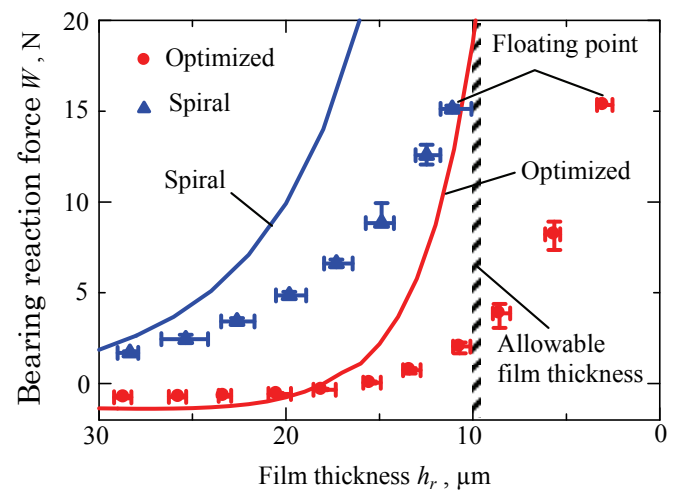

(a) Without temperature compensation

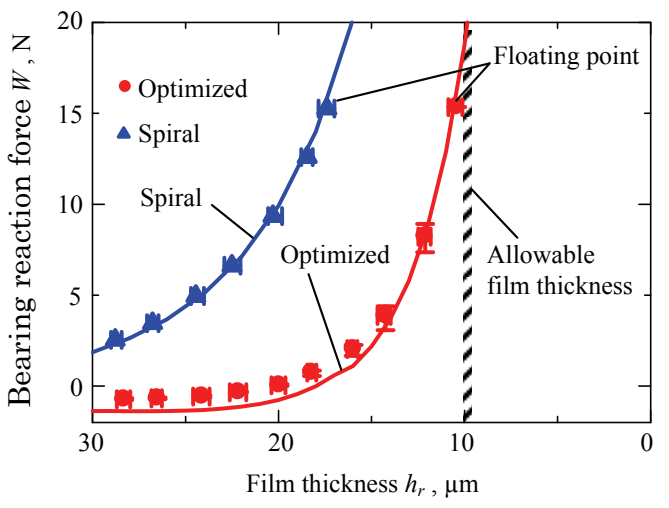

(b) With temperature compensation

Fig. 5 Relationship between film thickness and bearing reaction force, (a) Without temperature compensation, (b) With temperature compensation 
force and the air film thickness respectively. In the figure, the plots and the error bars show the mean value and the variability of measurements, and the solid lines show the theoretical results. The experimental repeatability was confirmed by measurements of more than five times. Figure 5(a) shows the results in the case of neglecting the temperature compensation, Fig. 5(b) shows the results in the case of considering the temperature compensation. In these figures, theoretical results are same values.

In the case of neglecting the temperature compensation, there is a difference between the experimental and theoretical results as shown in Fig. 5(a). On the other hand, fairly good agreement is shown between experimental and theoretical results with temperature compensation as shown in Fig. 5(b). As described above, the film thickness could be measured with high accuracy by applying proposed temperature compensation for the probe. Furthermore, both values of bearing reaction force of the optimized bearing and spiral grooved bearing are increased with a decrease in film thickness. Especially in the range from 10 to $15 \mu \mathrm{m}$, the value of the optimized bearing increases rapidly because of the effect of negative pressure on outer circumference. This leads to increase the bearing stiffness compared with the spiral grooved bearing. In addition, the minimum film thickness of the optimized bearing, which equilibrates the load and the bearing reaction force, is smaller than that of the spiral grooved bearing. However, the minimum film thickness of the optimized bearing exceeds the allowable film thickness of $10 \mu \mathrm{m}$ which is set as the constraint condition in optimum design [8].

\subsection{Results of impact response displacement}

Figures 6(a)-(c) show results of experiments, non-linear and linear analyses under external force of 60 $\mathrm{N}$ respectively. Result of liner analysis can be obtained by solving the following equation (3).

$$
m \ddot{z}+c \dot{z}+k z=f(t)
$$

where, $m$ : math of stator, $k$ : spring coefficient, $c$ : damping coefficient, $z$ : displacement, $f(t)$ : impact force. In the Eq. (3), spring and damping coefficients, $k$ and $c$, can be obtained by solving the Reynolds equation using the perturbation method [8]. On the other hand, the response displacement of non-linear analysis can be obtained by solving the equation of motion for the bearing and the Reynolds equation simultaneously using the Runge-Kutta method. In these figures, undershoot means the minimum distance between the rotor and the bearing, and overshoot means the maximum distance.

As can be seen in Fig. 6(a), the values of undershoot and overshoot of the optimized bearing are about half of the values of the spiral grooved bearing. Furthermore, the settling time of vibration of the optimized bearing is very shorter than that of the spiral grooved bearing. The reason why the result was obtained is that the optimized bearing has high spring and damping coefficients caused by reduction of film thickness compared with that of the spiral grooved bearing as shown in Fig. 5. Moreover, it is found that the result of the linear analysis shown in Fig. 6 (b) has great difference from the results of experiment. Especially, the values of undershoot and overshoot of the linear analysis are significantly higher than those of the experiment because the non-linearity of air film thickness is significant under thin film thickness conditions. On the other hand, the results of the non-linear analysis are obtained same tendency with the

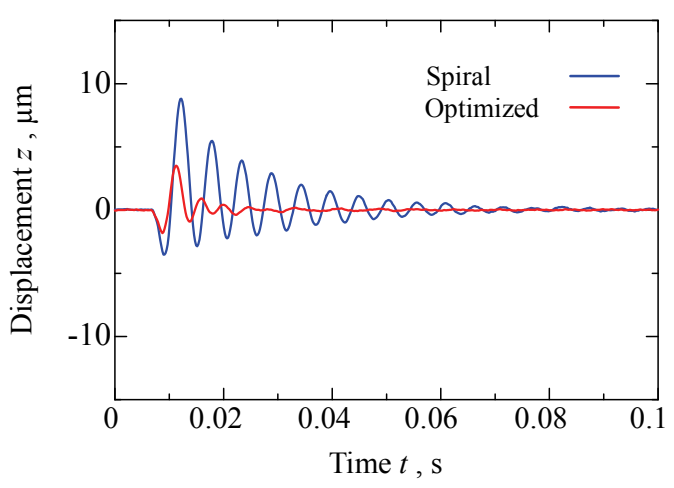

(a) Experimental results

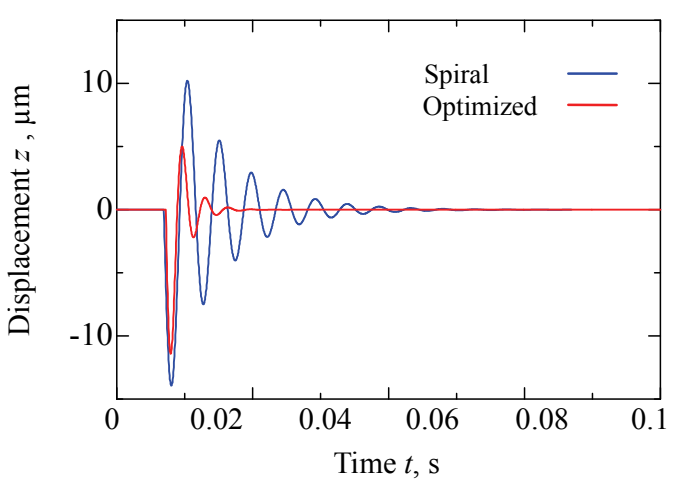

(b) Results of linear analysis

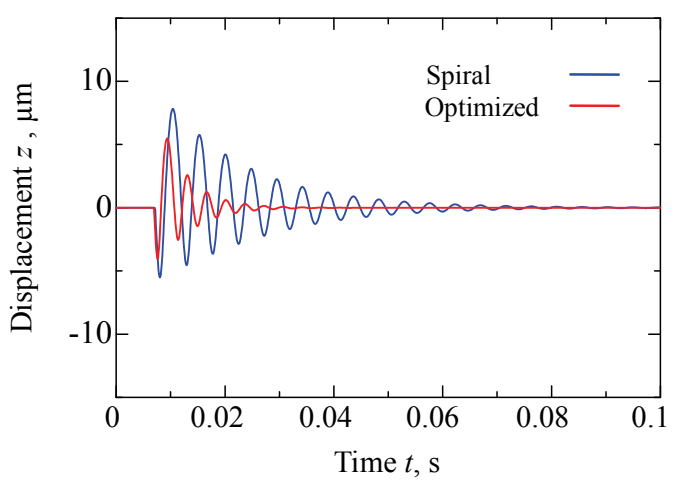

(c) Results of non-linear analysis

Fig. 6 Experimental and theoretical results of response displacement under external force of 60 [N], (a) Experimental results, (b) Results of linear analysis, (c) Results of non-linear analysis 


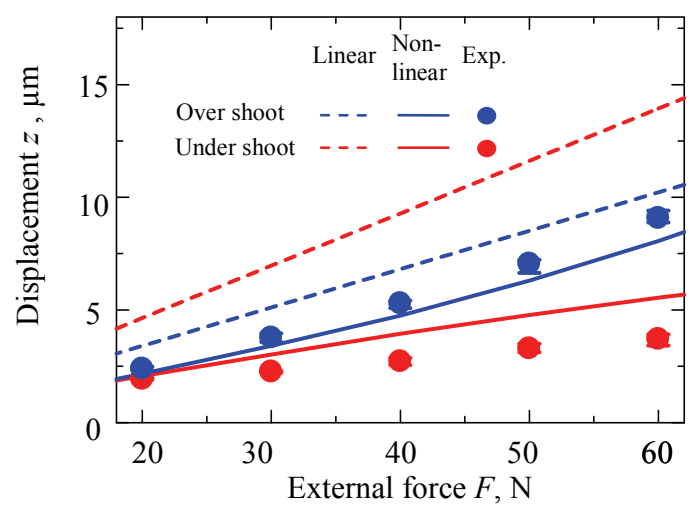

(a) Spiral grooved bearing

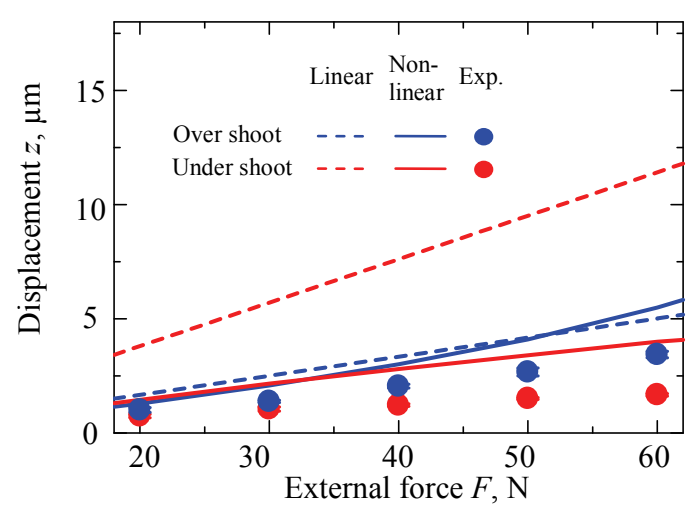

(b) Optimized bearing

Fig. 7 Relationship between response displacement and external force, (a) Spiral grooved bearing, (b) Optimized bearing

experimental results qualitatively and quantitatively. Consequently it was found that we should adopt the non-linear analysis to predict the vibration characteristics of the bearings. From above, it is confirmed that the optimized bearing has high impact resistance against the external impact force theoretically and experimentally.

Figure 7 shows the relationship between the external impact forces and the displacements of undershoot and overshoot. In addition, in these figures, plots show the experimental results, solid and dashed lines show the results of the non-linear and the linear analyses respectively. As can be seen in Fig. 7, values of the optimized bearing were lower than those of the spiral grooved bearing at the external force ranges from $20 \mathrm{~N}$ to $60 \mathrm{~N}$, similar to results shown in Fig. 6. In addition, fairly good agreements are shown qualitatively between the experiment and the non-linear analysis. However, the difference between these values of results of the optimized bearing is larger than those of the spiral grooved bearing. The reason why the difference was occurred is considered to be the effect of the misalignment of the rotor.

Figure 8 shows the relationship between the external force and the settling time, which is defined that the

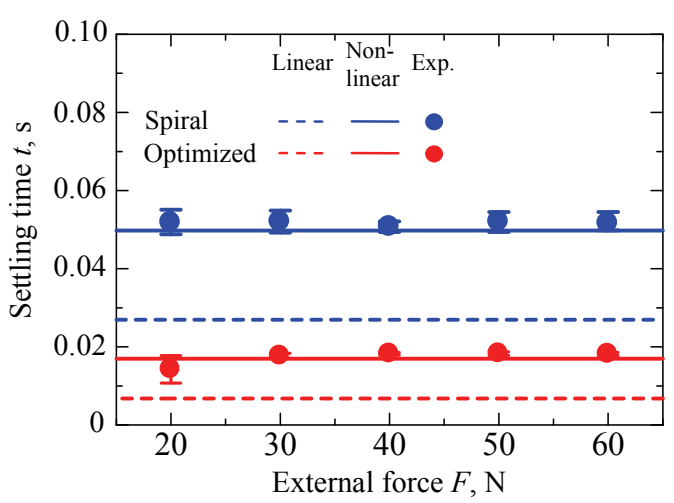

Fig. 8 Relationship between settling time and external force

response displacement is $\pm 5 \%$ or less of its maximum values. From the figure, the settling times of the optimized bearing are drastically shorter than those of the spiral grooved bearing within the external force from 20 $\mathrm{N}$ to $60 \mathrm{~N}$. As a result, it is confirmed that the optimized bearing has high damping property experimentally and theoretically. Moreover, fairly good agreements are shown between the experimental results and results of non-linear analysis.

For the purpose of the confirmation of resistance under higher impact force conditions which exceed the limitation of our test rig, we conducted the impact response analysis by non-liner theory under more high impact force condition of 140 N. Figure 9 shows the theoretical results of response displacement under external force of $140 \mathrm{~N}$. As can be seen in Fig. 9, there is possibility to occur self-excited vibration in the case of the spiral grooved bearing. On the other hand, the vibration amplitude of the optimized bearing decreases with time. As a result, it was found that the optimized bearing has high vibration characteristic compared with the spiral grooved bearing in a wide range of impact forces.

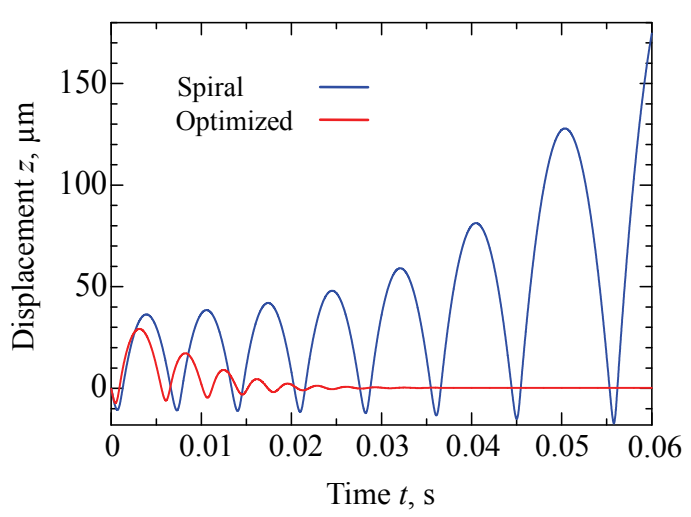

Fig. 9 Theoretical results of response displacement under external force of $140 \mathrm{~N}$ 


\section{Conclusions}

In this study, we carried out the impact experiments with the optimized bearing and the spiral grooved bearing and compared experimental results with theoretical results by linear and non-linear analyses. The conclusions are briefly summarized as follows.

We proposed the temperature compensation method for the eddy current proximity probe for high accuracy measurement of air film thickness. Experimental results of the bearing reaction force with the temperature compensation are good agreement with theoretical results.

Response displacement of the optimized bearing was about half of the value of the spiral grooved bearing, and the settling time of the optimized bearing was also significantly shorter than results of the spiral grooved bearing.

It was found that the non-linear analysis is required to predict the vibration characteristics of the hydrodynamic thrust air bearing.

It was confirmed experimentally and theoretically that the optimized bearing has high vibration characteristic compared with the spiral grooved bearing.

\section{References}

[1] Malanoski, S. B. and Pan, C. H. T., "The Static and Dynamic Characteristics of Spiral-Grooved Thrust Bearings," ASME Journal of Fluids Engineering, 87, 3, 1965, 547-555.

[2] James, D. D. and Potter, A. F., "Numerical Analysis of the Gas-Lubricated, Spiral-Groove Thrust Bearing Compressor," ASME Journal of Lubrication Technology, 89, 4, 1967, 439-443.

[3] Tatara, A., Koike, H. and Iwasaki, A., "Study of Gas Lubricated Spiral Groove Bearings:
Experimental Results of Pressure Distribution," Junkatsu (in Japanese), 16, 9, 1971, 680-686.

[4] Bonneau, D., Huitric, J. and Tournerie, B., "Finite Element Analysis of Grooved Gas Thrust Bearings and Grooved Gas Face Seals," ASME Journal of Tribology, 115, 3, 1993, 348-354.

[5] Hughes, S. J., Hogg, S. I. and Jones, T. V., "Analysis of a Gas-Lubricated Hydrodynamic Thrust Bearing," ASME Journal of Tribology, 118, 3, 1996 , 449-456.

[6] Hashimoto, H. and Ochiai, M., "Theoretical Analysis and Optimum Design of High Speed Gas Film Thrust Bearings (Static and Dynamic Characteristic Analysis with Experimental Verifications)," JSME Journal of Advanced Mechanical Design, Systems, and Manufacturing, 1, 1, 2007, 102-112.

[7] Hashimoto, H., Ochiai, M. and Nanba, T., "Theoretical Analysis and Optimum Design of High Speed Gas Film Thrust Bearings (Application to Optimum Design Problem)," JSME Journal of Advanced Mechanical Design, Systems, and Manufacturing, 1, 3, 2007, 306-318.

[8] Hashimoto, H. and Ochiai, M., "Optimization of Groove Geometry for Thrust Air Bearing to Maximize Bearing Stiffness," ASME Journal of Tribology, 130, 3, 2008, 1-11.

[9] Hashimoto, H., Tadashi, N., "Optimization of Groove Geometry for a Thrust Air Bearing According to Various Objective Functions," ASME Journal of Tribology, 131, 4, 2009, 1-10.

[10] Sunami, Y., et al., "Measurements of Film Thickness and Pressure Distribution for Optimized Thrust Air Bearing," Proceedings of the 2nd International Conference on Design Engineering and Science, ICDES2010, 2010, 223-228. 